\title{
Puerarin protects vascular smooth muscle cells from oxidized low-density lipoprotein-induced reductions in viability via inhibition of the p38 MAPK and JNK signaling pathways
}

\author{
YANWU HU ${ }^{1}$, HAITAO LI $I^{2}$, RUILI LI ${ }^{2}$, ZIJING WU ${ }^{1}$, WENXIN YANG ${ }^{1}$ and WEI QU ${ }^{3}$ \\ ${ }^{1}$ Department of Traditional Chinese Medicine, School of Medicine, Tonghua Normal University, Tonghua, Jilin 134002; \\ ${ }^{2}$ Department of Pharmacy, Affiliated Hospital, Changchun University of Chinese Medicine, Changchun, Jilin 130021; \\ ${ }^{3}$ Department of Pharmacy, Affiliated Jiangyin Hospital of Southeast University Medical College, \\ Jiangyin, Jiangsu 214400, P.R. China
}

Received April 24, 2020; Accepted August 19, 2020

DOI: $10.3892 /$ etm.2020.9400

\begin{abstract}
Puerarin belongs to the family of flavonoids extracted from Pueraria lobata (Wild.) Ohwi, which exhibits antioxidative, anti-inflammatory, anti-hyperglycemic, antitumor, anti-hypertensive and anti-atherosclerotic activities. In the present study, the effects of puerarin on oxidized low-density lipoprotein (ox-LDL)-stimulated vascular smooth muscle cells (VSMCs) were explored to understand the mechanisms underlying the anti-atherosclerotic effects of puerarin. VSMCs were treated with various concentrations of puerarin $(0,20,40$ and $80 \mu \mathrm{M})$ prior to stimulation with ox-LDL (50 $\mu \mathrm{g} / \mathrm{ml})$. VSMC viability was evaluated by performing MTT and Cell Counting Kit- 8 assays. Moreover, superoxide dismutase (SOD) and malondialdehyde (MDA) levels were measured by performing ELISAs. The mRNA expression levels of interleukin-6 (IL-6) and tumor necrosis factor- $\alpha$ (TNF- $\alpha$ ) were determined via reverse transcription-quantitative PCR. Western blotting was conducted to assess the levels of p38-MAPK and JNK phosphorylation. The results indicated that puerarin inhibited ox-LDL-induced VSMC viability. Moreover, puerarin significantly decreased the mRNA expression levels of IL-6 and TNF- $\alpha$, significantly reduced the production of MDA and significantly increased SOD activity in ox-LDL-stimulated VSMCs. Puerarin also inhibited ox-LDL-induced phosphorylation of p38 and JNK in VSMCs. The results suggested that puerarin reduced ox-LDL-induced VSMC viability via inhibition of the p38 MAPK and JNK signaling pathways. The present study provided theoretical evidence that puerarin may
\end{abstract}

Correspondence to: Professor Wei Qu, Department of Pharmacy, Affiliated Jiangyin Hospital of Southeast University Medical College, 163 Shoushan Road, Jiangyin, Jiangsu 214400, P.R. China E-mail: cpuquwei@163.com

Key words: puerarin, vascular smooth muscle cells, oxidized low-density lipoprotein, oxidative stress, inflammation, MAPK signaling pathway serve as a therapeutic agent to reduce the development of atherosclerosis.

\section{Introduction}

Atherosclerosis is an important pathological manifestation of cardiovascular diseases and chronic inflammatory disease (1), which is the result of a build-up of fatty materials, such as cholesterol and lipids (2-4). Atherosclerosis affects arterial blood vessels, resulting in thickening of the vascular wall and a narrowing of the lumen (5). Previous studies have indicated that cells of the arterial wall, including $\mathrm{T}$ cells, monocyte-derived macrophages, endothelial cells and vascular smooth muscle cells (VSMCs), are associated with the development of atherosclerosis $(1,6)$. Although the mechanism is not fully understood, it is widely accepted that the abnormal proliferation of VSMCs located in the arterial intima leads to intimal thickening of the aorta, serving an important role in the pathogenesis and progression of atherosclerosis $(7,8)$. Therefore, inhibiting VSMC proliferation may serve as a useful therapeutic approach for atherosclerosis.

Pueraria lobata (Wild.) Ohwi, is widely used in traditional Chinese medicine as a treatment for cardiovascular diseases, diabetes and liver diseases (9-11). Puerarin (4'-7'-dihy droxy-8- $\beta$-D-glucosylisoflavone, $\mathrm{C}_{21} \mathrm{H}_{20} \mathrm{O}_{10}$; Fig. 1), one of the major isoflavonoid compounds isolated from the root of Pueraria lobata, is considered as one of the main pharmacologically active constituents of this treatment (12). Moreover, puerarin has been reported to display various pharmacological properties, including anti-oxidative (13), anti-inflammatory (14), antitumor (15), anti-hypercholesterolemic (9), anti-hyperglycemic (16) and anti-hypertensive activities (13). Furthermore, puerarin has been reported to have beneficial effects in the treatment of atherosclerosis $(12,17,18)$. However, the role and mechanism underlying puerarin activity on oxidized low-density lipoprotein (ox-LDL)-stimulated VSMCs has not been previously reported. Ox-LDL-induced VSMC proliferation in the intima of the arterial wall serves a critical role in the progress of atherosclerosis (19), therefore inhibiting ox-LDL-induced proliferation may serve as a 
potential therapeutic strategy for atherosclerosis. The present study aimed to evaluate the potential protective effect of puerarin on ox-LDL-stimulated VSMCs and to further identify the underlying mechanisms of its action.

\section{Materials and methods}

Cell culture and treatments. Human VSMCs were obtained from The Cell Bank of Type Culture Collection of the Chinese Academy of Sciences. VSMCs were seeded $\left(1 \times 10^{4}\right.$ cells/well) into 96-well microplates and cultured in DMEM (Gibco; Thermo Fisher Scientific, Inc.) supplemented with 10\% heat-inactivated FBS (Hangzhou Sijiqing Biological Engineering Materials Co., Ltd.) and $1 \%$ penicillin/streptomycin (Sigma-Aldrich; Merck $\mathrm{KGaA}$ ) at $37^{\circ} \mathrm{C}$ with $5 \% \mathrm{CO}_{2}$. Following pretreatment with $0,20,40$ or $80 \mu \mathrm{M}$ puerarin (purity, $\geq 98 \%$; Sichuan Weikeqi Biological Technology Co., Ltd.) for $24 \mathrm{~h}$ at $37^{\circ} \mathrm{C}$, cells were treated with $50 \mu \mathrm{g} / \mathrm{ml}$ ox-LDL (Guangzhou Yiyuan Biological Technology Co., Ltd.) for $24 \mathrm{~h}$ at $37^{\circ} \mathrm{C}(20)$.

Cell viability assay. Cell viability was evaluated by performing an MTT assay (Beijing Solarbio Science \& Technology Co., Ltd.). Cells were seeded (1x104 cells/well) into 96-well microplates and cultured in DMEM overnight at $37^{\circ} \mathrm{C}$. Subsequently, cells were incubated with DMEM containing $0.01 \%$ DMSO or puerarin $(20,40$ or $80 \mu \mathrm{M})$ at $37^{\circ} \mathrm{C}$ for $24 \mathrm{~h}$. Cells were then stimulated with ox-LDL $(50 \mu \mathrm{g} / \mathrm{ml})$ for $24 \mathrm{~h}$ at $37^{\circ} \mathrm{C}$. Subsequently, $5 \mathrm{mg} / \mathrm{ml}$ MTT solution was added to each well for $4 \mathrm{~h}$ at $37^{\circ} \mathrm{C}$. DMSO $(150 \mu \mathrm{l})$ was added to each well for $15 \mathrm{~min}$ to dissolve the purple formazan. Absorbance was measured at a wavelength of $490 \mathrm{~nm}$ using a microplate reader (Thermo Fisher Scientific, Inc.).

The Cell Counting Kit-8 (CCK-8) assay (Sigma-Aldrich; Merck $\mathrm{KGaA}$ ) was conducted to determine cell viability according to the manufacturer's protocol. Briefly, following treatment, $10 \mu \mathrm{l} \mathrm{CCK}-8$ reagent was added into each well for $2 \mathrm{~h}$ at $37^{\circ} \mathrm{C}$. Absorbance was measured at a wavelength of $450 \mathrm{~nm}$ using a microplate reader (Thermo Fisher Scientific, Inc.). Cell viability was calculated according to the following formula: Cell viability $(\%)=$ absorbance of test sample/absorbance of control x100.

Detection of oxidative stress biomarkers, superoxide dismutase (SOD) and malondialdehyde (MDA). Cells were seeded $\left(1 \times 10^{4}\right.$ cells/well) into 6 -well microplates and cultured in DMEM overnight at $37^{\circ} \mathrm{C}$. Subsequently, cells were incubated with DMEM containing 0.01\% DMSO or puerarin (20, 40 and $80 \mu \mathrm{M}$ ) for $24 \mathrm{~h}$ at $37^{\circ} \mathrm{C}$. Cells were then stimulated with $50 \mu \mathrm{g} / \mathrm{ml}$ ox-LDL for $24 \mathrm{~h}$ at $37^{\circ} \mathrm{C}$. Cells were collected and lysed on ice using RIPA lysis buffer (Beyotime Institute of Biotechnology). Following centrifugation at $13,000 \mathrm{x} g$ for 5 min at $4^{\circ} \mathrm{C}$, the supernatant was collected to determine SOD (cat. no. 20190602) activity and MDA (cat. no. 20190508) content using commercial kits (Nanjing Jiancheng Institute of Biological Engineering Co., Ltd.) according to the manufacturer's protocol.

Western blotting. Cells were seeded $\left(1 \times 10^{4}\right.$ cells/well) into 6-well microplates and cultured in DMEM overnight at $37^{\circ} \mathrm{C}$.

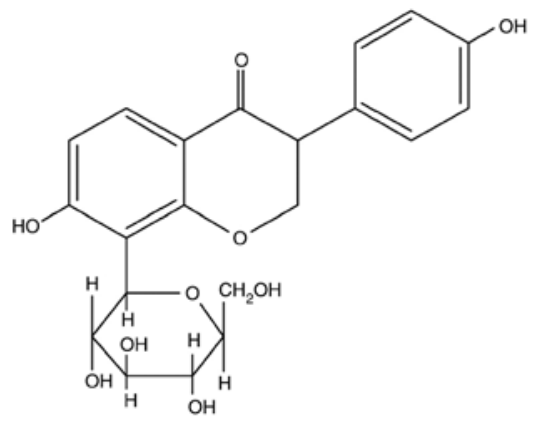

Figure 1. Chemical structure of puerarin.

Cells were incubated with DMEM containing 0.01\% DMSO or puerarin $(20,40$ and $80 \mu \mathrm{M})$ for $24 \mathrm{~h}$ at $37^{\circ} \mathrm{C}$. Cells were then stimulated with $50 \mu \mathrm{g} / \mathrm{ml}$ ox-LDL for $24 \mathrm{~h}$ at $37^{\circ} \mathrm{C}$. Cells were collected and lysed on ice with RIPA lysis buffer (Beyotime Institute of Biotechnology). Total protein was quantified using a bicinchoninic acid protein assay kit (Beyotime Institute of Biotechnology). Equal amounts of protein $(50 \mu \mathrm{g})$ were separated via $10 \%$ SDS-PAGE (120 V for $1.5 \mathrm{~h})$ and transferred onto PVDF membranes (100 V for $1 \mathrm{~h})$, which were blocked with 5\% skimmed milk in TBST $(0.05 \%$ Tween-20) for $1 \mathrm{~h}$ at room temperature. After blocking, the membranes were incubated overnight at $4^{\circ} \mathrm{C}$ with the following primary antibodies: Anti-p38 (1:1,000; cat. no. sc-7281; Santa Cruz Biotechnology, Inc.), anti-p-p38 (1:1,000; cat. no. sc-7937; Santa Cruz Biotechnology, Inc.), JNK (1:1,000; cat. no. sc-6531; Santa Cruz Biotechnology, Inc.), p-JNK (1:1,000; cat. no. sc-3824; Santa Cruz Biotechnology, Inc.), anti-GAPDH (1:1,000; cat. no. sc-6341; Santa Cruz Biotechnology, Inc.). Following incubation, the membranes were washed three times in TBST, before the membranes were incubated with an appropriate horseradish peroxidase-conjugated secondary antibody for $1 \mathrm{~h}$ at room temperature (1:5,000; cat. no. sc-5203; Santa Cruz Biotechnology, Inc.). Protein bands were visualized using enhanced chemiluminescence reagent (Amersham; Cytiva). Protein expression levels were semi-quantified using ImageJ software (version 1.46; National Institutes of Health) with GAPDH as the loading control.

Reverse transcription-quantitative PCR (RT-qPCR). Cells were seeded $\left(1 \times 10^{4}\right.$ cells/well) into 6-well microplates and cultured in DMEM overnight at $37^{\circ} \mathrm{C}$. Cells were incubated with DMEM containing $0.01 \%$ DMSO or puerarin $(20,40$ and $80 \mu \mathrm{M})$ for $24 \mathrm{~h}$ at $37^{\circ} \mathrm{C}$. Cells were then exposed to $50 \mu \mathrm{g} / \mathrm{ml}$ ox-LDL for $24 \mathrm{~h}$ at $37^{\circ} \mathrm{C}$. Subsequently, total RNA was extracted from the cells using TRIzol ${ }^{\circledR}$ (Takara Biomedical Technology Co., Ltd., China) according to the manufacturer's protocol. Total RNA was reverse transcribed using the PrimeScript RT Master Mix kit (Takara Biotechnology Co., Ltd.) at $37^{\circ} \mathrm{C}$ for $15 \mathrm{~min}$ and $85^{\circ} \mathrm{C}$ for $5 \mathrm{~min}$. Subsequently, qPCR was performed using SYBR ${ }^{\circledR}$ Premix Ex Taq (TransGen Biotech Co., Ltd.) with the following thermocycling conditions: Initial denaturation at $95^{\circ} \mathrm{C}$ for $5 \mathrm{~min}$; followed by 40 cycles of denaturation at $95^{\circ} \mathrm{C}$ for $10 \mathrm{sec}$, annealing at $60^{\circ} \mathrm{C}$ for $15 \mathrm{sec}$ and extension at $72^{\circ} \mathrm{C}$ for $30 \mathrm{sec}$; followed by a final extension at $72^{\circ} \mathrm{C}$ for $10 \mathrm{~min}$. The following primers were used: IL- 6 forward, 5'-CTCTCCGCAAGAGACTTCCA-3' and reverse, 

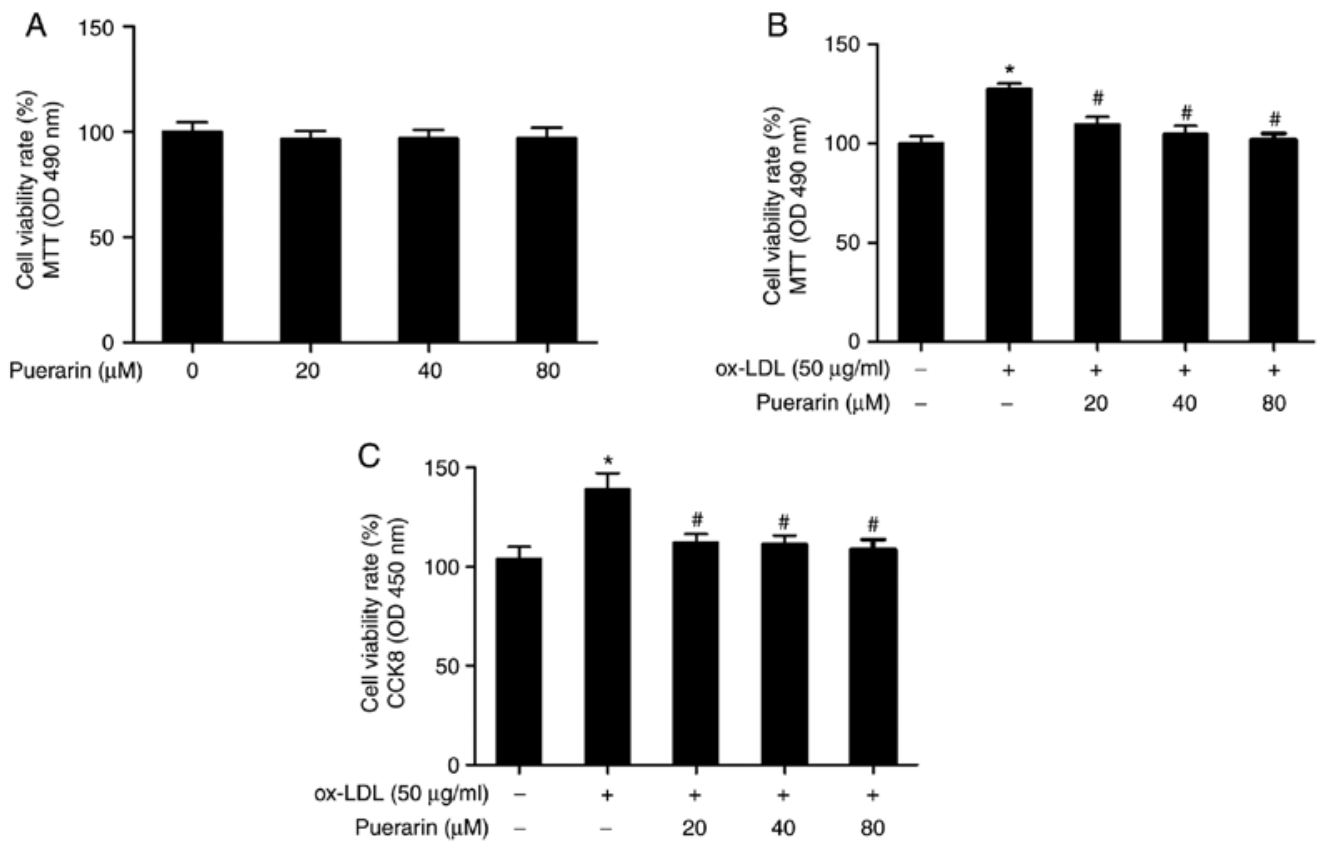

Figure 2. Puerarin inhibited cell viability in ox-LDL-stimulated VSMCs. The MTT assay was performed to assess the effect of puerarin on cell viability in (A) VSMCs and (B) ox-LDL-induced VSMCs. (C) The CCK- 8 assay was performed to assess the effect of puerarin on cell viability in VSMCs. "P $<0.05$ vs. control VSMCs; ${ }^{\text {P }}<0.05$ vs. ox-LDL-stimulated VSMCs. ox-LDL, oxidized low-density lipoprotein; VSMC, vascular smooth muscle cell; CCK-8, Cell Counting Kit-8; OD, optical density.
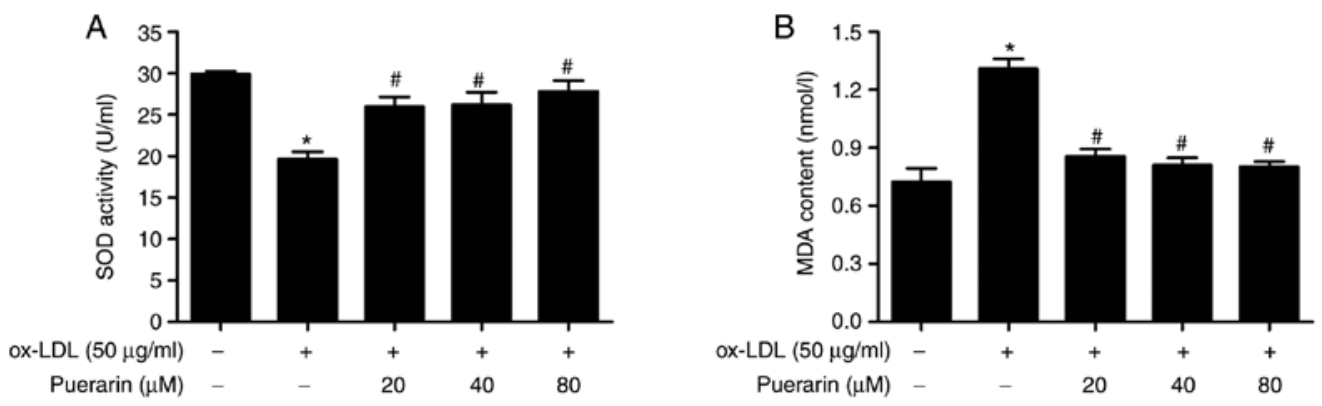

Figure 3. Puerarin regulates the production of oxidative stress-related makers in ox-LDL-induced VSMCs. Effect of puerarin on (A) SOD activity and (B) MDA content in ox-LDL-induced VSMCs. "P<0.05 vs. control VSMCs; ${ }^{*} \mathrm{P}<0.05$ vs. ox-LDL-stimulated VSMCs. ox-LDL, oxidized low-density lipoprotein; VSMC, vascular smooth muscle cell; SOD, superoxide dismutase; MDA, malondialdehyde.

5'-TGGTCTTCTGGAGTTCCGTT-3'; TNF- $\alpha$ forward, 5'-TCTCATCAGTTCTATGGCCC-3' and reverse, 5'-GGG AGTAGACAAGGTACAAC-3'; GAPDH forward, 5'-GTT ACCAGGGCTGCCTTCTC-3' and reverse, 5'-GATGGTGAT GGGTTTCCCGT-3'. mRNA expression levels were quantified using the $2^{-\Delta \Delta \mathrm{Cq}}$ method (21) and normalized to the internal reference gene GAPDH.

Statistical analysis. Statistical analyses were performed using SPSS (version 19.0; IBM Corp.). All experiments were performed in triplicate. Comparisons among multiple groups were analyzed using one-way ANOVA followed by Tukey's post hoc test. Data are presented as the mean \pm SEM. $\mathrm{P}<0.05$ was considered to indicate a statistically significant difference.

\section{Results}

Puerarin inhibits cell viability in ox-LDL-stimulated VSMCs. To evaluate the cytotoxicity of puerarin on VSMCs, cells were treated with puerarin $(0,20,40$ and $80 \mu \mathrm{M})$ for $24 \mathrm{~h}$. The MTT assay results suggested that puerarin did not significantly alter VSMC viability compared with the control group (Fig. 2A). Following incubation with different concentrations of puerarin for $24 \mathrm{~h}$, cells were stimulated with $50 \mu \mathrm{g} / \mathrm{ml}$ ox-LDL for $24 \mathrm{~h}$ to evaluate the effect of puerarin on cell viability in ox-LDL-induced VSMCs. Cell viability was significantly increased in ox-LDL-induced VSMCs compared with the control group (Fig. 2B). However, pretreatment with puerarin significantly inhibited ox-LDL-induced cell viability. Similar results were observed in the CCK-8 assay (Fig. 2C).

Puerarin regulates the production of oxidative stress-related markers in ox-LDL-stimulated VSMCs. To investigate the mechanism underlying the inhibitory effect of puerarin on ox-LDL-induced cell proliferation, the effect of puerarin on SOD activity and MDA content was determined. ox-LDL significantly increased the content of MDA in VSMCs compared with the control group (Fig. 3B). Puerarin 

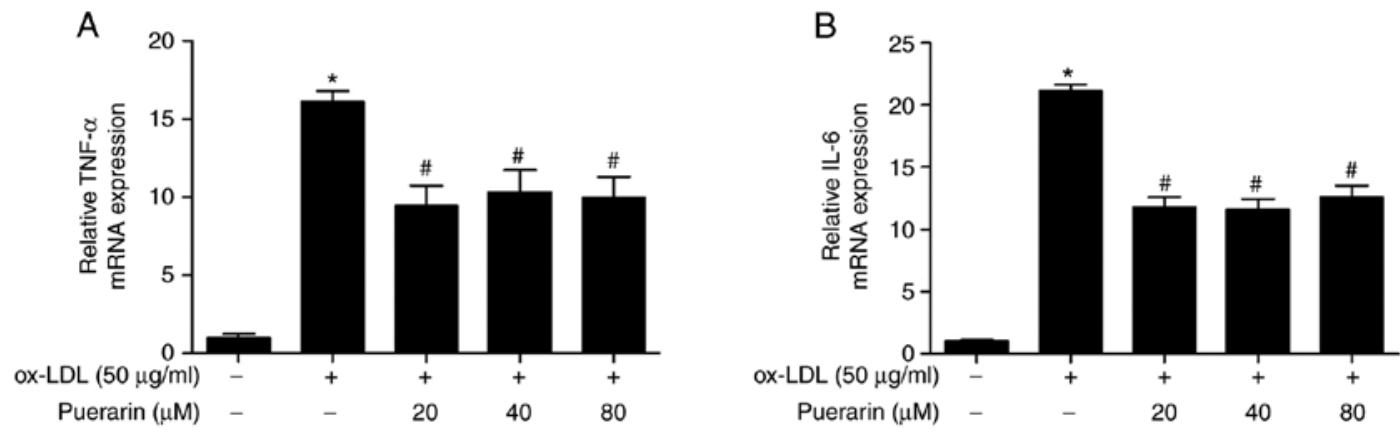

Figure 4. Puerarin inhibits proinflammatory cytokines in ox-LDL-induced VSMCs. Effect of puerarin on the mRNA expression levels of (A) TNF- $\alpha$ and (B) IL-6 in ox-LDL-induced VSMCs. "P<0.05 vs. control VSMCs; "P<0.05 vs. ox-LDL-stimulated VSMCs. ox-LDL, oxidized low-density lipoprotein; VSMC, vascular smooth muscle cell.
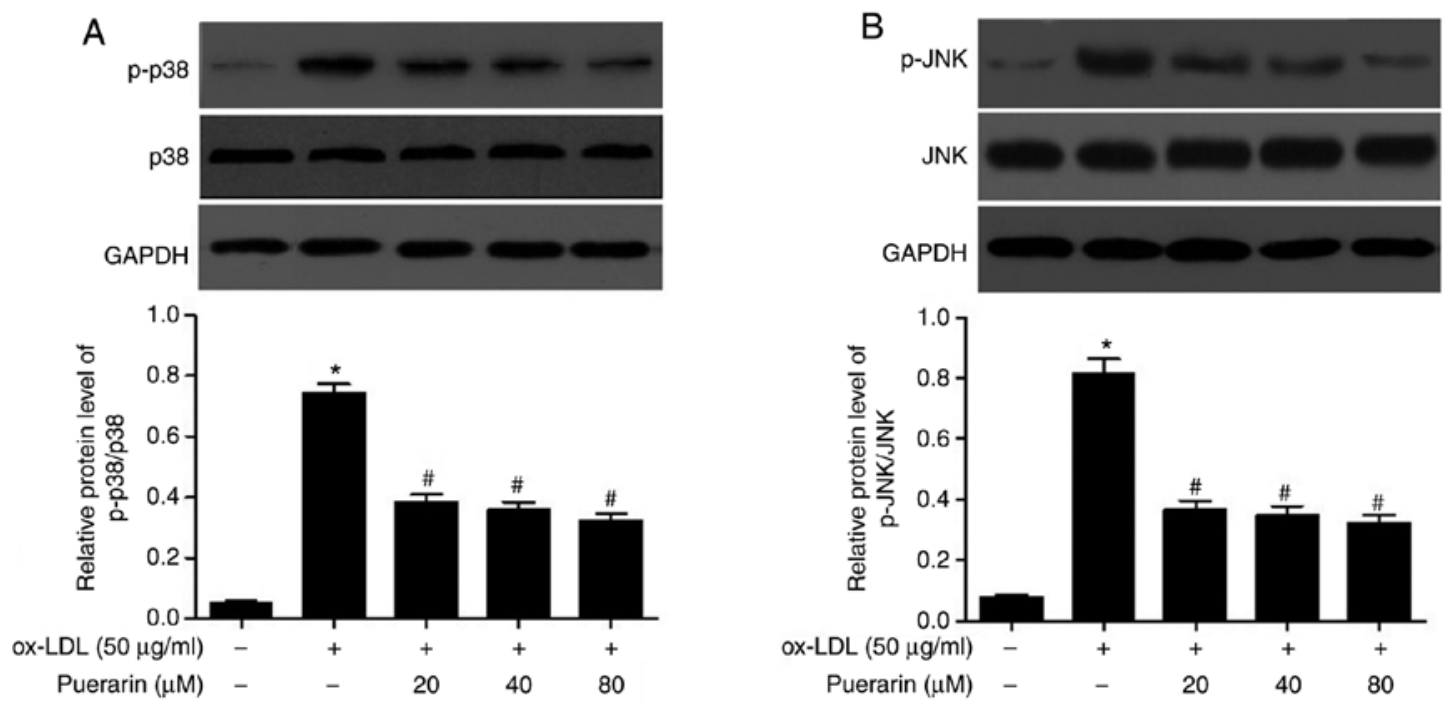

Figure 5. Puerarin prevents the activation of $\mathrm{p} 38$ MAPK and JNK signaling pathways in ox-LDL-induced VSMCs. The protein expression ratios of (A) p-38/p38 and (B) p-JNK/JNK were determined via western blotting. " $\mathrm{P}<0.05$ vs. control VSMCs; ${ }^{\#} \mathrm{P}<0.05$ vs. ox-LDL-stimulated VSMCs. ox-LDL, oxidized low-density lipoprotein; VSMC, vascular smooth muscle cell; p, phosphorylated.

pretreatment significantly attenuated ox-LDL-induced MDA levels. In addition, compared with the control group, SOD activity was significantly decreased by ox-LDL in VSMCs, but pretreatment with puerarin diminished ox-LDL-mediated effects on SOD activity (Fig. 3B). The results suggested that puerarin significantly inhibited ox-LDL-induced oxidative stress in VSMCs.

Puerarin inhibits proinflammatory cytokines in ox-LDL-stimulated VSMCs. The effect of puerarin on IL-6 and TNF- $\alpha$ mRNA expression levels in ox-LDL-stimulated VSMCs was examined. ox-LDL significantly increased the mRNA expression levels of IL-6 and TNF- $\alpha$ in VSMCs compared with the control group (Fig. 4A). However, puerarin pretreatment significantly decreased the mRNA expression levels of IL-6 and TNF- $\alpha$ in ox-LDL-stimulated VSMCs (Fig. 4B). The results suggested that puerarin inhibited proinflammatory cytokines in ox-LDL-stimulated VSMCs.

Puerarin prevents the activation of $p 38$ MAPK and JNK signaling pathways in ox-LDL-stimulated VSMCs. To further elucidate whether the p38 MAPK and JNK signaling pathways were involved in the effect of puerarin in ox-LDL-stimulated VSMCs, the phosphorylation levels of p38 and JNK were analyzed via western blotting. The expression levels of p-p38 and p-JNK were significantly increased by ox-LDL in VSMCs compared with the control group (Fig. 5A and B). Puerarin pretreatment significantly decreased the expression levels of p-p38 and p-JNK in ox-LDL-stimulated VSMCs. The results indicated that puerarin inhibited the activation of p38 MAPK and JNK signaling pathways in ox-LDL-induced VSMCs.

\section{Discussion}

During the progression of atherosclerosis, abnormal VSMC proliferation and migration serve key roles in causing stenosis and intimal thickening (22). A number of factors affect VSMC proliferation and migration, including hypertension, dyslipidemia and oxidative stress (4). As a well-established index of oxidative stress, ox-LDL is involved in the generation of atherosclerotic lesions (4). Numerous studies have indicated that ox-LDL can induce VSMC proliferation and migration, which are pathological events that are crucial for neointima 
formation (23) and stimulate activation of the MAPK signaling pathway $(24,25)$. Therefore, if a substance can effectively prevent ox-LDL-induced VSMC proliferation, it should inhibit the development of atherosclerosis.

Puerarin, a major isoflavone isolated from the root of Pueraria lobata, has been reported to treat various cardiovascular diseases, including arterial hypertension, angina, myocardial infarction and arrhythmia (26-28). Puerarin has anti-atherosclerotic activity in diet-induced atherosclerosis in rabbits and rats $(17,18)$. Puerarin prevents the pathogenesis of atherosclerosis, which may be mediated by its anti-inflammatory and antioxidative stress actions $(28,29)$. A key finding of the present study was that puerarin significantly inhibited ox-LDL-induced VSMC viability. In the present study, whether cell viability was inhibited by puerarin when VSMCs were exposed to ox-LDL was investigated. The results demonstrated that ox-LDL enhanced VSMC viability compared with the control group, but pretreatment with puerarin significantly reversed ox-LDL-mediated effects in VSMCs. Moreover, compared with the control group, ox-LDL significantly decreased SOD activity and significantly increased MDA content, suggesting that the level of oxidative stress in VSMCs induced by ox-LDL was higher compared with control conditions. The antioxidant activity of puerarin was further indicated by its ability to reverse ox-LDL-mediated alterations in SOD activity and MDA content.

For the clinical development of puerarin as a treatment, understanding the mechanism underlying how it inhibits VSMC proliferation is important. The present study focused on the MAPK signaling pathway in VSMCs. Previous studies demonstrated that ox-LDL activated the MAPK signaling pathway, including p38, ERK and JNK in VSMCs, which promoted cell proliferation $(30,31)$. Puerarin prevents ox-LDL-induced proliferation and inhibits the phosphorylation of ERK1/2 in VSMCs (32). Puerarin inhibits VSMC proliferation induced by fine particulate matter via reducing the elevated expression levels of p-p38 MAPK (33). Moreover, puerarin markedly stimulates bone marrow stromal cell differentiation towards an osteogenic phenotype via the ERK1/2 and p38-MAPK signaling pathways (34). The aforementioned study results indicated that puerarin displays protective effects in different diseases by regulating MAPK signaling pathways. In the present study, puerarin inhibited the activation of the p38 MAPK and JNK signaling pathways in ox-LDL-induced VSMCs.

Certain studies have speculated that the p38 MAPK signaling pathway is associated with inflammasome activation $(5,35,36)$. Activation of the p38 MAPK signaling pathway increases the expression of TNF- $\alpha$ and IL-6 $(36,37)$. In the present study, similar results were obtained. Although the present study did not further investigate the mechanism of MAPK signaling pathway and inflammasome activation, relative indices were observed and it was suggested that the MAPK signaling pathway was involved in the activation of inflammation in ox-LDL-induced VSMCs. The results of the present study indicated that the phosphorylation levels of p38 and JNK were significantly increased in ox-LDL-induced VSMCs compared with the control group. Similarly, the mRNA expression levels of IL- 6 and TNF- $\alpha$ were significantly increased in ox-LDL-induced VSMCs compared with control VSMCs. However, puerarin significantly downregulated the expression levels of p-p38 and p-JNK and reduced the mRNA expression levels of IL- 6 and TNF- $\alpha$ in ox-LDL-stimulated VSMCs. The effect of puerarin on IL-6 and TNF- $\alpha$ did not display a concentration gradient trend, which indicated that the effect of puerarin was not closely related to the concentration. The effect of puerarin on other proinflammatory cytokines requires further investigation. The results of the present study suggested that alterations in proinflammatory cytokines were closely related to alterations in the expression levels of p-p38 MAPK and p-JNK, indicating that puerarin altered the expression of TNF- $\alpha$ and IL- 6 by regulating the p38 MAPK and JNK signaling pathways in ox-LDL-induced VSMCs. However, cell networks are complex, thus, whether puerarin exerts its function via other signaling pathways in ox-LDL-induced VSMCs requires further investigation.

In conclusion, the results of the present study suggest that puerarin inhibited ox-LDL-induced VSMC viability, and that the antiproliferative effects of puerarin were partly associated with inactivation of the p-p38 MAPK and p-JNK signaling pathways, which was mediated via suppression of the expression levels of TNF- $\alpha$ and IL-6. Therefore, the results of the present study suggest that puerarin may inhibit the development of atherosclerosis.

\section{Acknowledgements}

Not applicable.

\section{Funding}

The present study was supported by the Science and Technology Research Project of the Education Department of Jilin Province (grant no. JJKH20191098KJ) and the Science And Technology Project of Traditional Chinese Medicine of Jilin Administration of Traditional Chinese Medicine (grant no. 2020135).

\section{Availability of data and materials}

The datasets used and/or analysed during the current study are available from the corresponding author on reasonable request.

\section{Authors' contributions}

YH performed the experiments and wrote the manuscript. RL, ZW and WY performed the cell experiments. HL conceived the study and revised the manuscript. WQ designed the study and analysed the data. All authors read and approved the final manuscript.

\section{Ethics approval and consent to participate}

Not applicable.

\section{Patient consent for publication}

Not applicable. 


\section{Competing interests}

The authors declare that they have no competing interests.

\section{References}

1. Liu J, Ren Y, Kang L and Zhang L: Oxidized low-density lipoprotein increases the proliferation and migration of human coronary artery smooth muscle cells through the upregulation of osteopontin. Int J Mol Med 33: 1341-1347, 2014.

2. Dell'omo G, Penno G, Pucci L, Lucchesi D, Fotino C, Del Prato S and Pedrinelli R: ACE gene insertion/deletion polymorphism modulates capillary permeability in hypertension. Clin Sci (Lond) 111: 357-364, 2006.

3. Zhu F, Li C, Jin XP, Weng SX, Fan LL, Zheng Z, Li WL, Wang F, Wang WF, Hu XF, et al: Celastrol may have an anti-atherosclerosis effect in a rabbit experimental carotid atherosclerosis model. Int J Clin Exp Med 7: 1684-1691, 2014.

4. Liu Z, Ren X, Yang Z, Zhao Y, Ji L, Li J and Yue W: Effects and mechanisms of indol-2,3-dione on atherosclerosis. Int J Clin Exp Med 7: 2087-2091, 2014.

5. Hu Y, Sun B, Liu K, Yan M, Zhang Y, Miao C and Ren L: Icariin attenuates high-cholesterol diet induced atherosclerosis in rats by inhibition of inflammatory response and p38 MAPK signaling pathway. Inflammation 39: 228-236, 2016.

6. González-Navarro $\mathrm{H}$, Abu Nabah YN, Vinué A Andrés-Manzano MJ, Collado M, Serrano M and Andrés V: p19(ARF) deficiency reduces macrophage and vascular smooth muscle cell apoptosis and aggravates atherosclerosis. J Am Coll Cardiol 55: 2258-2268, 2010.

7. Daemen MJ, Lombardi DM, Bosman FT and Schwartz SM: Angiotensin II induces smooth muscle cell proliferation in the normal and injured rat arterial wall. Circ Res 68: 450-456, 1991.

8. Ross R: The pathogenesis of atherosclerosis: A perspective for the 1990s. Nature 362: 801-809, 1993

9. Yan LP, Chan SW, Chan AS, Chen SL, Ma XJ and Xu HX Puerarin decreases serum total cholesterol and enhances thoracic aorta endothelial nitric oxide synthase expression in diet-induced hypercholesterolemic rats. Life Sci 79: 324-330, 2006

10. Fu J, Jing W, Wang W, Chen S, Zhang J and Liu A: A novel and effective chromatographic approach to the separation of isoflavone derivatives from Pueraria lobata. Molecules 20: 4238-4253, 2015.

11. Zhu X, Xie M, Wang K, Zhang K, Gao Y, Zhu L and Zhou F: The effect of puerarin against IL-1 $\beta$-mediated leukostasis and apoptosis in retinal capillary endothelial cells (TR-iBRB2). Mol Vis 20: 1815-1823, 2014.

12. Bao MH, Zhang YW, Lou XY, Xiao Y, Cheng Y and Zhou HH: Puerarin protects endothelial cells from oxidized low density lipoprotein induced injuries via the suppression of LOX-1 and induction of eNOS. Can J Physiol Pharmacol 92: 299-306, 2014.

13. Meng XH, Ni C, Zhu L, Shen YL, Wang LL and Chen YY: Puerarin protects against high glucose-induced acute vascular dysfunction: Role of heme oxygenase-1 in rat thoracic aorta Vascul Pharmacol 50: 110-115, 2009.

14. Yang X, Hu W, Zhang Q, Wang Y and Sun L: Puerarin inhibits C-reactive protein expression via suppression of nuclear factor kappaB activation in lipopolysaccharide-induced peripheral blood mononuclear cells of patients with stable angina pectoris. Basic Clin Pharmacol Toxicol 107: 637-642, 2010.

15. Hien TT, Kim HG, Han EH, Kang KW and Jeong HG Molecular mechanism of suppression of MDR1 by puerarin from Pueraria lobata via NF-kappaB pathway and cAMP-responsive element transcriptional activity-dependent up-regulation of AMP-activated protein kinase in breast cancer MCF-7/adr cells. Mol Nutr Food Res 54: 918-928, 2010.

16. Hsu FL, Liu IM, Kuo DH, Chen WC, Su HC and Cheng JT: Antihyperglycemic effect of puerarin in streptozotocin-induced diabetic rats. J Nat Prod 66: 788-792, 2003.

17. Bao L, Zhang Y, Wei G, Wang Y, Ma R, Cheng R, Ren X and Agula B: The anti-atherosclerotic effects of puerarin on induced-atherosclerosis in rabbits. Biomed Pap Med Fac Univ Palacky Olomouc Czech Repub 159: 53-59, 2015.

18. Fu R, Zhang Y, Guo Y, Xu Y and Chen F: Digital gene expression analysis of the pathogenesis and therapeutic mechanisms of ligustrazine and puerarin in rat atherosclerosis. Gene 552: 75-80, 2014.
19. Chang WC, Yu YM, Chiang SY and Tseng CY: Ellagic acid suppresses oxidised low-density lipoprotein-induced aortic smooth muscle cell proliferation: Studies on the activation of extracellular signal-regulated kinase $1 / 2$ and proliferating cell nuclear antigen expression. Br J Nutr 99: 709-714, 2008.

20. Hu Y, Liu K, Yan M, Zhang Y, Wang Y and Ren L: Icariin inhibits oxidized low-density lipoprotein-induced proliferation of vascular smooth muscle cells by suppressing activation of extracellular signal-regulated kinase $1 / 2$ and expression of proliferating cell nuclear antigen. Mol Med Rep 13: 2899-2903, 2016.

21. Livak KJ and Schmittgen TD: Analysis of relative gene expression data using real-time quantitative PCR and the 2(-Delta Delta C(T)) method. Methods 25: 402-408, 2001.

22. Yasunari K, Kohno M, Kano H, Hanehira T, Minami M and Yoshikawa J: Anti-atherosclerotic action of vascular D1 receptors. Clin Exp Pharmacol Physiol Suppl 26: S36-S40, 1999.

23. Witztum JL and Steinberg D: The oxidative modification hypothesis of atherosclerosis: Does it hold for humans? Trends Cardiovasc Med 11: 93-102, 2001.

24. Guo LL, Chen YJ, Wang T, An J, Wang CN, Shen YC, Yang T, Zhao L, Zuo QN, Zhang XH, et al: Ox-LDL-induced TGF- $\beta 1$ production in human alveolar epithelial cells: Involvement of the Ras/ERK/PLTP pathway. J Cell Physiol 227: 3185-3191, 2012.

25. Liao L, Zhou Q, Song Y, Wu W, Yu H, Wang S, Chen Y, Ye M and Lu L: Ceramide mediates Ox-LDL-induced human vascular smooth muscle cell calcification via p38 mitogen-activated protein kinase signaling. PLoS One 8: e82379, 2013.

26. Prasain JK, Peng N, Rajbhandari R and Wyss JM: The Chinese Pueraria root extract (Pueraria lobata) ameliorates impaired glucose and lipid metabolism in obese mice. Phytomedicine 20: 17-23, 2012.

27. Fu C, Chen B, Jin X, Liu X, Wang F, Guo R, Chen Z, Zheng H, Wang $\mathrm{L}$ and Zhang Y: Puerarin protects endothelial progenitor cells from damage of angiotensin II via activation of ERK1/2Nrf2 signaling pathway. Mol Med Rep 17: 3877-3883, 2018.

28. Gao Y, Wang X and He C: An isoflavonoid-enriched extract from Pueraria lobata (kudzu) root protects human umbilical vein endothelial cells against oxidative stress induced apoptosis. J Ethnopharmacol 193: 524-530, 2016.

29. Ji L, Du Q, Li Y and Hu W: Puerarin inhibits the inflammatory response in atherosclerosis via modulation of the NF-kappaB pathway in a rabbit model. Pharmacol Rep 68: 1054-1059, 2016.

30. Hu YW, Li HT, Liu K, Yan MT, Zhang Y and Ren LQ: Effects of icariin on proliferation of vascular smooth muscle cell induced by ox-LDL via impacting MAPK signaling pathway. Zhongguo Zhong Yao Za Zhi 41: 3655-3660, 2016 (In Chinese).

31. Li W, Zhi W, Zhao J, Yao Q, Liu F and Niu X: Cinnamaldehyde protects VSMCs against ox-LDL-induced proliferation and migration through $\mathrm{S}$ arrest and inhibition of p38, JNK/MAPKs and NF- $\mathrm{kB}$. Vascul Pharmacol 108: 57-66, 2018.

32. Hu Y, Liu K, Bo S, Yan M,Zhang Y, Miao C and Ren L: Inhibitory effect of puerarin on vascular smooth muscle cells proliferation induced by oxidised low-density lipoprotein via suppressing ERK 1/2 phosphorylation and PCNA expression. Pharmazie 71: 89-93, 2016.

33. Wan Q, Liu Z and Yang Y: Puerarin inhibits vascular smooth muscle cells proliferation induced by fine particulate matter via suppressing of the p38 MAPK signaling pathway. BMC Complement Altern Med 18: 146, 2018.

34. Yang X, Yang Y, Zhou S, Gong X, Dai Q, Zhang P and Jiang L: Puerarin stimulates osteogenic differentiation and bone formation through the ERK1/2 and p38-MAPK signaling pathways. Curr Mol Med 17: 488-496, 2018.

35. Wang M, Li Z, Zhang X, Xie X, Zhang Y, Wang X and Hou Y: Rosuvastatin attenuates atrial structural remodelling in rats with myocardial infarction through the inhibition of the p38 MAPK signalling pathway. Heart Lung Circ 24: 386-394, 2015.

36. Craig R, Larkin A, Mingo AM, Thuerauf DJ, Andrews C, McDonough PM and Glembotski CC: p38 MAPK and NF-kappa $\mathrm{B}$ collaborate to induce interleukin-6 gene expression and release. Evidence for a cytoprotective autocrine signaling pathway in a cardiac myocyte model system. J Biol Chem 275: 23814-23824, 2000.

37. Yang C, Liu W, Shan H, Yu X, Zhang X, Zeng B and Qian Y: Naringin inhibits titanium particles-induced up-regulation of TNF- $\alpha$ and IL-6 via the p38 MAPK pathway in fibroblasts from hip periprosthetic membrane. Connect Tissue Res: 1-10, 2020. 